\title{
DESEMPENHO DE IDOSOS COM E SEM DECLÍNIO COGNITIVO LEVE NA VERSÃO REDUZIDA DO TESTE WCST-64
}

Cristiane Silva Esteves

Instituto Federal de Educação, Ciência e Tecnologia do Rio Grande do Sul (IFRS)

Camila Rosa Oliveira

Instituto Meridional IMED Passo Fundo

Valéria Gonzatti

Pontifícia Universidade Católica do Rio Grande do Sul (PUCRS)

Manuela Polidoro Lima

Pontifícia Universidade Católica do Rio Grande do Sul (PUCRS)

Carmen Moret Tatay

Universidad Católica de Valência San Vicente Mártir

Irani Iracema de Lima Argimon Pontifícia Universidade Católica do Rio Grande do Sul (PUCRS)

Tatiana Quarti Irigaray

Pontifícia Universidade Católica do Rio Grande do Sul (PUCRS)

\begin{abstract}
Resumo
Declínio Cognitivo Leve ( $D C L$ ) é uma condição clínica que apresenta perdas cognitivas acima do esperado para idade ou escolaridade, não preenchendo critérios diagnósticos para quadros demenciais. Este artigo comparou o desempenho de 56 idosos no Teste Wisconsin de Classificação de Cartas, versão reduzida (WCST-64) em uma amostra de idosos com e sem $\mathrm{DCL}$ ( $\mathrm{n}=28$ por grupo), emparelhados por idade e escolaridade (média de idade $=68,93 ; \mathrm{DP}=5,06$; escolaridade média $=13,21 ; \mathrm{DP}=5,06)$. Os instrumentos utilizados foram questionário sociodemográfico, bateria de testes neurocognitivos e de capacidade funcional e o WCST-64. Os dados foram analisados utilizando análises descritivas e inferenciais. O grupo com $\mathrm{DCL}$ obteve pior resultado apenas no escore número de erros do WCST-64 $(p=0,029)$. Os resultados sugerem que o WCST-64 apresenta potencial para diferenciar o desempenho de idosos com e sem DCL, o que possibilita a utilização desse instrumento como ferramenta de rastreio e de auxílio no processo diagnóstico dessa população.
\end{abstract}

Palavras-chave: idosos; cognição; desempenho; testes neuropsicológicos. 


\title{
PERFORMANCE OF ELDERLY WITH AND WITHOUT MILD COGNITIVE IMPAIRMENT IN THE WCST-64 TEST
}

\begin{abstract}
Mild Cognitive Impairment (MCI) is a clinical condition which presents greater cognitive loss than expected for their age or education, do not meeting diagnostic criteria for dementia cases. This article compared the performance of elderly in the reduced version of the Wisconsin Card Sorting Test (WCST-64) in a sample of 56 elderly with an without MCI ( $n=28$ per group) matched by age and by education (mean age $=68.93 ; S D=5.06$; education mean $=13.21 ; S D=5.06)$. The instruments applied were: a sociodemographic questionnaire, a battery of neurocognitive tests and functional capacity, and WCST-64. Data were analyzed through descriptive and inferential analyzes. The DCL group had a worse result only in the WCST-64 error score $(p=0.029)$. The results suggest that the WCST-64 has the potential to distinguish the performance of adults with and without MCI, indicating the possibility of using this instrument as a screening tool and as a support of this population diagnostic process.

Keywords: elderly people; cognition; performance; neuropsychological tests.
\end{abstract}

\section{RENDIMIENTO DE ANCIANOS CON Y SIN DETERIORO COGNITIVO LEVE EN LA PRUEBA WCST-64}

\begin{abstract}
Resumen
El deterioro cognitivo leve $(D C L)$ es una condición clínica que presenta pérdidas cognitivas por encima de lo esperado para la edad o la escolaridad, no cumpliendo criterios diagnósticos para los cuadros demencia. El presente artículo comparó el desempeño de ancianos en la prueba Wisconsin de clasificación de cartas versión reducida (WCST-64) en una muestra de 56 ancianos con y sin DCL ( $n=28$ por grupo), emparejados por edad y por escolaridad (media de años de edad $=68,93 ; \mathrm{DP}=5,06$; media de años de estudio $=13,21 ; \mathrm{DP}=5,06)$. Los instrumentos utilizados fueron: cuestionario sociodemográfico, batería de pruebas neurocognitivas y de capacidad funcional y el WCST-64. Los datos fueron analizados por medio de análisis descriptivos e inferenciales. El grupo con DCL obtuvo peor resultado sólo en la puntuación número de errores del WCST-64 $(p=0,029)$. Los resultados indican que el WCST-64 presenta potencial para diferenciar el desempeño de adultos con y sin $\mathrm{DCL}$, posibilitando la utilización de ese instrumento como herramienta de rastreo y de auxilio en el proceso diagnóstico de esa población.
\end{abstract}

Palabras clave: ancianos; cognición; desempeño; instrumentos neuropsicológicos.

\section{INTRODUÇÃO}

O aumento significativo no número da população de idosos é considerado um fenômeno global (Kreuz \& Franco, 2017; Slongo, Albrecht, Lavouras, Esteves, \& Barcelos, 2009). Há uma reestruturação constante das pirâmides etárias devido à contínua queda da taxa de nascimentos, aos avanços da medicina preventiva e ao aumento da expectativa de vida (Ribeiro, 2008). Ao mesmo tempo em que cresce o número total de idosos, aumentam as queixas e os problemas de memória, além das demandas por atendimento nos ambulatórios e serviços de saúde (Kreuz \& Franco, 2017; Veras, 2009). Diante dessa realidade, pesquisadores têm se interessado pelos diferentes aspectos do envelhecimento humano, desenvolvendo uma multiplicidade de estudos sobre a velhice.

Devido ao aumento da expectativa de vida no mundo, torna-se necessário pensar, também, na qualidade com que se viverão esses anos a mais. Com isso, considera-se que o prolongamento da vida poderá ser considerado, de fato, como 
conquista, no momento em que se agregar qualidade de vida a esses anos adicionais (Gomes \& Othero, 2016; Veras, 2009). Por esse motivo, há uma preocupação com o modo de vida dos idosos e com os aspectos de vulnerabilidade que levam a um aumento da velocidade do declínio das habilidades cognitivas (Ribeiro, Oliveira, Cupertino, Neri, \& Yassuda, 2010).

O envelhecimento normal pode vir acompanhado de uma diminuição na evocação da memória episódica e na capacidade de inibição, além de lentificação no processamento de informação e de redução da flexibilidade cognitiva (Green, 2000; Silva, 2017). Considera-se natural que, durante o envelhecimento, os idosos apresentem déficits das funções cognitivas (Lopes, Bastos, \& Argimon, 2017), entretanto, em um grau que não venha a prejudicar a vida cotidiana do indivíduo e nem a comprometer a sua funcionalidade (Argimon, 2006; Argimon \& Stein, 2005; Ribeiro et al., 2010). Diante disso, entende-se que as mudanças que ocorrem no cérebro dos idosos cognitivamente saudáveis não influenciam, ou fazem pouca diferença, no seu funcionamento diário (Papalia \& Olds, 2013. Os autores referidos ainda salientam que a maior parte dos idosos apresenta boa saúde física e mental, principalmente, nos primeiros anos da velhice.

O declínio das habilidades cognitivas é influenciado pela idade e, também, por aspectos genéticos, sociodemográficos e de estilo de vida (Charchat-Fichman, Caramelli, Sameshima, \& Nitrini, 2005; Ribeiro et al., 2010). O Declínio Cognitivo Leve ( $D C L$ ) é considerado uma condição clínica em que o indivíduo demonstra perdas cognitivas maiores do que o esperado para sua escolaridade e idade, mas que essa condição não preencha critérios diagnósticos para demência (Gauthier et al., 2006; Knopman, Boeve, \& Petersen, 2003; Petersen et al., 1999; Petersen et al., 2001). Winblad et al. (2004) modificaram os critérios para diagnosticar a presença de $\mathrm{DCL}$, por constatarem a evolução para o quadro de demência em pacientes que apresentavam não só déficits de memória, mas, também, queixas/déficits em outros domínios cognitivos (Petersen et al., 2014). Os critérios sugeridos por Winblad et al. (2004) para DCL, que serão os adotados no presente estudo, são: a) queixas subjetivas de declínio em qualquer domínio cognitivo, preferencialmente, corroboradas por um informante; b) ausência de demência (de acordo com os critérios do Manual Diagnóstico e Estatístico de Transtornos Mentais - DSM-5) (American Psychological Association - APA, 2014); c) independência para realizar as atividades de vida diária e d) comprometimento objetivo em qualquer domínio cognitivo (mensurado por meio de testes).

No DCL, as alterações de natureza executiva estão presentes de forma precoce e quantitativamente intensa (Banhato \& Nascimento, 2007). As funções executivas podem ser afetadas também pelo processo de envelhecimento normal e estão relacionadas à capacidade do indivíduo de realizar ações independentes e voluntárias, auto-organizadas e voltadas para metas específicas (Lopes et al., 2017; Sullivan, Riccio, \& Castillo, 2009). Integradas, as funções executivas auxiliam no processo de tomada de decisão, na avaliação e adequação de 
estratégias e nos comportamentos para resolução de problemas (Malloy-Diniz, Lasmar, Gazinelli, Fuentes, \& Salgado, 2007; Silva, 2017). Além disso, auxiliam na realização de ações de forma adaptativa, permitindo mudanças rápidas e flexíveis do comportamento frente a novas exigências do ambiente (Diamond, 2013).

Há, na literatura, um predomínio de pesquisas sobre a memória em idosos em detrimento das demais funções cognitivas, tais como as funções executivas, as quais podem permitir um conhecimento diferenciado a respeito do DCL. Diante dessa realidade, o presente artigo se propõe a comparar o desempenho de idosos com e sem DCL no Teste Wisconsin de Classificação de Cartas, versão reduzida (WCST-64), que avalia as funções executivas, para verificar a sua possibilidade de utilização como um instrumento de rastreio de DCL.

\section{MÉTODO}

\section{Delineamento}

Trata-se de um estudo transversal quantitativo e comparativo.

\section{Participantes}

A amostra inicial contou com 111 idosos, com idade igual ou superior a 60 anos, da região metropolitana de Porto Alegre, Rio Grande do Sul, Brasil.

Para recrutamento dos participantes utilizou-se a técnica de amostragem por conveniência, por meio de anúncio no jornal e por meio da técnica bola de neve (Penrod, Preston, Cain, \& Starks, 2003). Esta é uma técnica de amostragem não probabilística, na qual os participantes iniciais de uma pesquisa indicam novos participantes que, por sua vez, indicam outros participantes, e assim, sucessivamente, até que seja alcançado o objetivo do estudo (Baldin e Munhoz, 2011).

Foram excluídos da amostra nove indivíduos que não terminaram a aplicação dos instrumentos; um que apresentou problemas sensórios primários (auditivo e visual) não corrigidos durante o momento da avaliação, e sete que obtiveram escores no Mini Exame do Estado Mental (MEEM; adaptado por Chaves \& Izquerdo, 1992) abaixo dos seguintes pontos de corte, indicando provável quadro demencial: 22 pontos para 5 anos de escolaridade; 23 para escolaridade entre 6 e 11 anos e 24 para os indivíduos com 12 anos ou mais de estudo (Kochhann, Varela, Lisboa, \& Chaves, 2010). Optou-se por essa classificação, pois foi desenvolvida a partir de um estudo de normatização realizado com idosos no sul do Brasil. A amostra final desta pesquisa contou com 56 participantes.

$\mathrm{Na}$ determinação dos grupos, foram seguidos os critérios sugeridos por Winblad et al. (2004) para identificar a presença de DCL. Dessa forma, foram incluídos no presente estudo instrumentos que pudessem também avaliar queixas cognitivas subjetivas, comprometimento cognitivo objetivo, independência para 
realizar as atividades de vida diária e ausência de sinais sugestivos de demência, de acordo com os critérios do DSM-5 (APA, 2014).

Os idosos foram classificados como possuindo $\mathrm{DCL}$ no momento em que apresentaram escore $z$ menor ou igual a $-1,5$ em pelo menos uma das medidas de avaliação dos seguintes domínios cognitivos: atenção/velocidade de processamento, memória, funções executivas e linguagem. A habilidade visuoconstrutiva foi medida por meio da cópia da figura do MEEM, sendo considerado prejudicado pontuação equivalente a 0 (zero). A partir da análise do desempenho nos instrumentos, identificaram-se os indivíduos com DCL $(n=43)$ e sem DCL $(n=51)$. Dentre esses participantes, foi possível realizar o emparelhamento quanto à escolaridade e à idade de 28 idosos com $\mathrm{DCL}$ em relação a 28 idosos sem DCL.

\section{Instrumentos}

a) Ficha de Dados Sociodemográficos para caracterização da amostra e investigação das variáveis idade, anos de estudo, sexo, estado civil, coabitação e percepção de saúde.

b) Mini Exame do Estado Mental (MEEM) que é composto por questões que avaliam orientação para tempo, orientação para local, registro de três palavras, atenção e cálculo, lembrança de três palavras, linguagem e habilidade visuoconstrutiva (por meio do subteste cópia de figura) (Chaves \& Izquerdo, 1992).

c) Subteste Dígitos (Ordem Direta e Indireta) da Escala Wechsler de Inteligência para Adultos, terceira edição (WAIS-III) (Nascimento, 2000; Wechsler, 1997) que compreende duas tarefas diferentes com dígitos, sendo a primeira na ordem direta e a segunda na ordem indireta. Segundo Cunha (2000), essas tarefas avaliam atenção, memória imediata (dígitos na ordem direta), memória e capacidade de reversibilidade (dígitos na ordem inversa).

d) Trail Making Test (TMT) que fornece medidas de flexibilidade cognitiva, atenção dividida e velocidade de processamento visual. O participante precisa ligar números e letras, que estão dentro de círculos. O critério de correção utilizado é o tempo e, quanto maior ele for, pior é o desempenho (Strauss, Sherman, \& Spreen, 2006).

e) Teste de Aprendizagem Auditivo-Verbal de Rey - RAVLT (Malloy-Diniz et al., 2007; Rey, 1958) que é um teste simples e rápido de administrar e seu uso tem sido amplamente reconhecido na área de neuropsicologia. Fornece uma medida de memória recente (aprendizagem verbal), retenção de informações depois de um período de tempo no qual outras atividades são realizadas e a memória de reconhecimento.

f) Teste Wisconsin de Classificação de Cartas - 64 cartas (WCST-64) (Kongs, Thompson, Iverson, \& Heaton, 2000) que mede as funções executivas, exigindo do participante a capacidade para desenvolver e manter uma estratégia apropriada 
de solução de problema por meio de condições de estímulos mutáveis a fim de atingir uma meta futura. Avalia o raciocínio abstrato e a capacidade para gerar estratégias de solução de problemas, investigando os componentes de flexibilidade cognitiva, inibição e resolução de problemas não verbais. Os escores foram calculados por meio de software específico desenvolvido para a correção do WCST64 (Heaton \& Par, 2000), sendo consideradas as pontuações das seguintes categorias: número de erros, respostas perseverativas, erros perseverativos, erros não perseverativos, número de respostas de nível conceitual, total de categorias completas, tentativas para formar a primeira categoria, rupturas e índice "aprendendo a aprender".

g) Teste de Fluência Verbal Fonêmica - FAS (Strauss et al., 2006) que é uma tarefa de fluência verbal fonêmica que envolve a listagem do maior número de palavras possíveis em um minuto para letras específicas. Avalia flexibilidade cognitiva e busca lexical.

h) Teste de Fluência Verbal Semântica - Categoria Animais (Strauss et al., 2006) que é uma tarefa de fluência verbal semântica que verifica a capacidade de organizar o pensamento e as estratégias utilizadas para a busca de palavras. 0 teste consiste em solicitar ao participante que diga o maior número possível de nomes de diferentes animais em um minuto.

i) Subteste Vocabulário da Escala Wechsler de Inteligência para Adultos, terceira edição (WAIS-III) (Nascimento, 2000; Wechsler, 1997) que é uma tarefa com características de produção verbal livre que avalia linguagem e formação de conceitos.

j) Escala de Katz (Lino, Pereira, Camacho, Ribeiro Filho, \& Buksman, 2008) que é usada para a avaliação das atividades de vida diária em idosos. O grau de assistência exigida é avaliado em seis atividades: tomar banho, vestir-se, ir ao banheiro, transferência, continência e alimentar-se. A Escala de Katz foi aplicada no formato Likert que pontua cada item de 0 a 3 , sendo que a pontuação 0 representa independência completa; 1 está relacionada com o uso de ajuda não humana (acessórios como bengalas, barras, apoio em móveis); 2 relaciona-se à ajuda humana e a pontuação 3, uma completa dependência.

\section{Procedimentos}

\section{Coleta dos Dados}

Este estudo foi aprovado pelo Comitê de Ética em Pesquisa da Pontifícia Universidade Católica do Rio Grande do Sul (CAAE no 14769713.1.0000.5336). Todos os participantes assinaram o Termo de Consentimento Livre e Esclarecido antes do início das avaliações. A avaliação foi realizada em local apropriado, durante uma sessão, de forma individual, com duração média de 90 minutos. 
Análise dos dados

Análises descritivas, como média, desvio-padrão e percentuais foram utilizadas a fim de descrever as características sociodemográficas, clínicas e de desempenho dos dois grupos (com e sem DCL) no WCST-64. A normalidade dos dados foi verificada por meio do Teste Kolmogorov-Smirnov, do Teste de ShapiroWilks e pelo coeficiente de assimetria (skewness), não sendo satisfeitos os pressupostos para técnicas paramétricas, além do número de participantes desse estudo. Em vista disso, as comparações entre os dois grupos foram realizadas por meio do $U$ de Mann-Whitney. O Teste Qui-quadrado foi realizado para comparação das variáveis nominais. O pacote estatístico utilizado foi o SPSS versão 20.0 para Windows. Consideraram-se resultados significativos quando $p \leq 0,05$.

\section{RESULTADOS}

Em relação à amostra geral $(n=56)$, as idades variaram de 60 a 80 anos $(M=68,89 ; D P=4,86)$ e os anos de estudo de 4 a 22 anos $(M=13,05 ; D P=$ $4,33)$, sendo $82 \%(n=46)$ mulheres. A Tabela 1 mostra a comparação dos dados sociodemográficos e clínicos dos grupos com e sem DCL. O estado civil mais frequente foi o casado(a) ou com companheiro(a) em ambos os grupos, assim como a percepção subjetiva de saúde foi classificada como boa pela maioria dos participantes dos grupos. Os dois grupos não apresentaram diferenças significativas nas demais variáveis avaliadas.

Tabela 1.

Dados sociodemográficos e clínicos entre os grupos com e sem $\mathrm{DCL}$

\begin{tabular}{|c|c|c|c|c|c|c|}
\hline & \multicolumn{2}{|c|}{$\begin{array}{l}\text { Sem DCL } \\
(n=28)\end{array}$} & \multicolumn{2}{|c|}{$\begin{array}{l}\text { Com DCL } \\
(n=28)\end{array}$} & \multirow[t]{2}{*}{$U / X^{2}$} & \multirow[t]{2}{*}{$p$} \\
\hline & $M$ & $D P$ & $M$ & $D P$ & & \\
\hline Idade (anos) & 68,93 & 5,06 & 68,86 & 4,74 & 379 & $0,831^{A}$ \\
\hline Escolaridade (anos) & 13,21 & 4,25 & 12,89 & 4,48 & 390 & $0,974^{A}$ \\
\hline MEEM & 28,21 & 1,77 & 27,21 & 1,99 & 275 & $0,052^{A}$ \\
\hline GDS-15 & 2,43 & 2,49 & 3,32 & 3,84 & 375 & $0,775^{A}$ \\
\hline Sexo $(\%)$ & & & & & 0,487 & $0,485^{\mathrm{B}}$ \\
\hline Mulheres & 24 & $(86)$ & 22 & $(79)$ & & \\
\hline Homens & 4 & $(14)$ & 6 & $(21)$ & & \\
\hline Estado Civil (\%) & & & & & 5,459 & $0,141^{B}$ \\
\hline Casado(a) & 13 & $(46)$ & 10 & $(36)$ & & \\
\hline Viúvo(a) & 8 & $(29)$ & 8 & $(28)$ & & \\
\hline Separado(a) & 3 & $(11)$ & 9 & $(32)$ & & \\
\hline Solteiro(a) & 4 & $(14)$ & 1 & (4) & & \\
\hline Coabitação (\%) & & & & & 5,540 & $0,236^{\mathrm{B}}$ \\
\hline
\end{tabular}




$\begin{array}{ccccccc}\text { Sozinho(a) } & 10 & (36) & 14 & (50) & & \\ \text { Esposo(a)/companheiro(a) } & 10 & (36) & 6 & (22) & \\ \begin{array}{c}\text { Esposo(a)/companheiro(a) e } \\ \text { filhos(as)/netos(as) }\end{array} & 4 & (14) & 4 & (14) & & \\ \text { Filhos(as)/netos(as) } & 4 & (14) & 2 & (7) & & \\ \text { Outros } & 0 & (0) & 2 & (7) & & \\ \text { Percepção da saúde (\%) } & & & & & 1,900 & 0,593^{\mathrm{B}} \\ \quad \text { Boa } & 24 & (86) & 22 & (77) & & \\ \quad \text { Regular } & 4 & (14) & 5 & (18) & & \\ \text { Ruim } & 0 & (0) & 1 & (4) & \end{array}$

Nota. MEEM = Mini Exame do Estado Mental. GDS-15 = Escala de Depressão Geriátrica, versão reduzida. ${ }^{A}=$ Teste $U$ de Mann-Whitney. ${ }^{B}=$ Teste Qui-quadrado.

De acordo com a Tabela 2 , os dois grupos diferenciaram-se significativamente apenas no escore quantidade de erros do WCST-64, sendo que os idosos com DCL obtiveram pior desempenho nessa categoria. Diante de tal resultado, pode-se entender que os idosos com DCL, em comparação aos sem $\mathrm{DCL}$, cometeram maior quantidade de erros, embora não tenha havido diferença entre os tipos de erros (perseverativos ou não perseverativos).

Tabela 2.

Comparação do desempenho dos grupos com e sem DCL no WCST-64

\begin{tabular}{|c|c|c|c|c|c|c|}
\hline & \multicolumn{2}{|c|}{$\begin{array}{l}\text { Sem DCL } \\
(n=28)\end{array}$} & \multicolumn{2}{|c|}{$\begin{array}{l}\text { Com DCL } \\
(n=28)\end{array}$} & \multirow[t]{2}{*}{$U$} & \multirow[t]{2}{*}{$p$} \\
\hline & $M$ & $D P$ & $M$ & $D P$ & & \\
\hline \multicolumn{7}{|l|}{ WCST-64 } \\
\hline Total de erros & 25,14 & 9,28 & 31,18 & 10,73 & 259 & 0,029 \\
\hline Respostas perseverativas & 14,14 & 8,33 & 20,68 & 14,13 & 298 & 0,123 \\
\hline Erros perseverativos & 12,11 & 6,14 & 17,14 & 10,44 & 298 & 0,123 \\
\hline Erros não perseverativos & 13,04 & 7,74 & 14,04 & 9,13 & 372 & 0,743 \\
\hline Respostas de nível conceitual & 30,68 & 12,91 & 23,25 & 13,95 & 276 & 0,056 \\
\hline Categorias completadas & 1,86 & 1,21 & 1,43 & 1,23 & 316 & 0,199 \\
\hline $\begin{array}{c}\text { Tentativas para formar primeira } \\
\text { categoria }\end{array}$ & 19,36 & 17,34 & 32,54 & 24,98 & 297 & 0,115 \\
\hline Falhas em manter o contexto & 0,75 & 1,01 & 0,68 & 0,91 & 381 & 0,834 \\
\hline Aprendendo a aprender & $-13,71$ & 12,93 & $-18,99$ & 15,31 & 51 & 0,379 \\
\hline
\end{tabular}


Nota. WCST-64 = Teste Wisconsin de Classificação de Cartas, versão reduzida.

\section{DISCUSSÃO}

O presente artigo visou comparar o desempenho de idosos com e sem DCL no WCST-64, verificando se o WCST-64 pode ser considerado como um possível instrumento para o rastreio de $\mathrm{DCL}$, ampliando a compreensão sobre idosos com DCL (em uma perspectiva das funções executivas). Os resultados encontrados revelaram que o grupo com DCL obteve pior desempenho no WCST-64, quando comparado ao grupo sem $\mathrm{DCL}$, na quantidade de erros cometidos, demonstrando, assim, maior dificuldade na realização da tarefa. O maior número de erros cometidos pelo grupo com prejuízo cognitivo pode indicar dificuldade para identificação de estratégias que sejam mais adequadas para solução de um problema, diante de uma diversidade de estímulos. Embora não tenha sido encontrada diferença estatisticamente significativa entre os grupos quanto aos tipos de erros, observa-se que o grupo com DCL apresentou média mais elevada do que o grupo sem DCL nos erros perseverativos, sugerindo que o grupo com DCL parece ter perseverado em um tipo de combinação de cartas, não utilizando o feedback do instrutor para buscar novas soluções para o problema.

Sendo assim, tal dado sugere uma limitação desse grupo em relação às suas percepções para outros modos de resolução mais eficientes, pois, mesmo com a indicação de erro dada pelo aplicador do teste, esses idosos permaneceram com o mesmo padrão de respostas inadequadas, demonstrando um possível prejuízo nas funções executivas. Lopes, Ziemniczak, Nascimento e Argimon (2015) entendem que o funcionamento executivo é responsável pela continuidade dos comportamentos e pelo comprometimento com o objetivo desejado, pois possibilita que a pessoa aproveite os estímulos externos, provenientes do meio ambiente, e as suas experiências anteriores para que consiga focar na meta a ser alcançada. Isso leva a inferir que os idosos com DCL apresentaram déficits nas funções executivas. Outros estudos também sugerem que indivíduos com o diagnóstico de DCL demonstram alterações nas funções executivas em uma mesma proporção que apresentam déficits na memória episódica (Chen, Ratcliff, \& Belle, 2001; Griffith et al., 2003; Royall \& Chiodo, 2004).

O estudo de Hamdan e Bueno (2005) comparou o desempenho de idosos com e sem DCL no WCST e encontrou diferença significativa entre o total de categorias completas, entretanto não encontrou essa diferença no total de erros. Uma hipótese para não ter sido detectada essa diferença entre os idosos com e sem DCL em relação ao total de erros pode ser o fato de que a amostra do estudo citado foi composta por idosos em geral, diferente da amostra do presente estudo que foi pareada, ou seja, apresentou-se mais homogênea e semelhante em relação às seguintes variáveis que podem ser confundidoras no resultado do teste como a escolaridade e a idade. Já a pesquisa realizada por Delano-Wood et al. (2008) com 
idosos com DCL demonstrou que, mesmo após o controle das variáveis idade, escolaridade e nível de depressão, foi constatado prejuízos nas funções executivas, o que corrobora com o presente estudo que, após o emparelhamento da amostra, também constatou diferenças de desempenho entre os grupos. No estudo de Leon, Hahn-Ketter, Carrion e Pliskin (2014), o diagnóstico de DCL foi um preditor para o desempenho no WCST (categorias completadas).

Além disso, o desempenho no teste demonstrou ser afetado pela escolaridade dos participantes. No estudo de Ballesteros, Mayas e Reales (2013), idosos com DCL também apresentaram pior desempenho no WCST (percentual de erros, respostas perseverativas, erros perseverativos e erros não perseverativos) quando comparados aos grupos de adultos jovens e de idosos saudáveis, além de pior desempenho em velocidade de processamento. Idosos com DCL e Alzheimer apresentam menor ativação na região frontal do cérebro em comparação a idosos saudáveis, podendo esse comportamento estar associado ao pior desempenho nas funções executivas (Chen et al., 2009). Corroborando com esta afirmativa, o estudo de Satler, Guimarães e Tomaz (2017) apontou que idosos com Alzheimer apresentaram maior dificuldade na habilidade de resolução de problemas, quando comparados com idosos cognitivamente saudáveis.

No grupo com DCL observou-se tendência em apresentar menor número de respostas de nível conceitual, sugerindo maior dificuldade na memória operacional. Durante o envelhecimento normal, é esperado que os idosos apresentem um declínio gradual das funções cognitivas (Lopes et al., 2015; 2017). Uma das hipóteses que explica as mudanças cognitivas é a diminuição do funcionamento das funções executivas (Braver \& West, 2008; Silva, 2017). O prejuízo das funções executivas pode ser considerado como um marcador do diagnóstico diferencial entre o envelhecimento normal e a demência (Carlson, Xue, Zhou, \& Fried, 2009; Mcguinness, Barrett, Craig, Lawson, \& Passmore, 2010). Baseando-se nisso e no resultado obtido no WCST-64, onde o grupo com DCL apresentou desempenho significativamente pior do que o grupo sem $\mathrm{DCL}$, pode-se inferir que os resultados desse teste permitem um diagnóstico diferencial quanto à presença ou não de DCL.

Entretanto, o WCST é um teste multifatorial, por esse motivo, o prejuízo no seu desempenho pode se dar por variadas razões, nem todas relacionadas especificamente às funções executivas (Strauss et al., 2006). Além disso, diversas funções cognitivas são necessárias para se responder ao WCST, influenciando, também, seus resultados (Kaland, Smith, \& Mortensen, 2008), o que mostra a importância de ser realizada uma avaliação cognitiva que englobe todas as funções cognitivas e não somente um teste. Diante disso, sugere-se que o WCST-64 possa ser utilizado como um teste de rastreio de DCL. 


\section{CONSIDERAÇÕES FINAIS}

O presente estudo identificou pior desempenho no teste WCST-64 pelos idosos com DCL, quando comparados com os sem essa condição, em apenas um dos escores desse instrumento (número de erros). Com isso, entende-se que os indivíduos com DCL demonstraram dificuldade de modificar um estilo de resposta considerada imprópria no teste e de inibir essa tendência comportamental mais imediata em prol de uma resposta mais adequada e elaborada. Tal processo pode também servir como padrão de respostas destes sujeitos nas situações adversas da vida diária. Considera-se uma limitação do presente estudo o tamanho da amostra e a técnica utilizada para amostragem, pois não favorecem a generalização dos resultados obtidos. Entretanto, devido à importância dos dados obtidos no presente estudo referentes à possibilidade da utilização do WCST-64 como instrumento de rastreio para o DCL, sugere-se que novos estudos sejam realizados com uma amostra maior, visando aprofundar os dados encontrados. Conforme apontado anteriormente, o WCST-64 demonstrou ser um teste que permite a diferenciação entre idosos normais e com DCL. Contudo, ainda são necessários estudos que busquem estabelecer dados normativos de referência para a população idosa, a fim de auxiliar no processo diagnóstico de DCL e de demais transtornos neurocognitivos.

\section{DECLARAÇÃO DE CONFLITO DE INTERESSES}

Não há conflito de interesses.

\section{REFERÊNCIAS}

American Psychological Association (APA) (2014). Manual de Diagnóstico e Estatístico de Transtornos Mentais DSM-5. Porto Alegre, RS: Artmed.

Argimon, I. I. L. (2006). Aspectos cognitivos em idosos. Avaliação Psicológica, $5(2), 243-245$.

Argimon, I. I. L., Bicca, M., Timm, L. A., \& Vivan, A. (2006). Funções executivas e a avaliação de flexibilidade de pensamento em idosos. Revista Brasileira de Ciência e Envelhecimento Humano, 3(2), 35-42. doi:10.5335/rbceh.2012.84

Argimon, I. I. L., \& Stein, L. M. (2005). Habilidades cognitivas em indivíduos muito idosos: Um estudo longitudinal. Caderno de Saúde Pública, 21(1), 64-72. doi: 10.1590/S0102-311X2005000100008

Baldin, N., Munhoz, E. M. B. (2011). Snowball (bola de neve): Uma técnica metodológica para pesquisa em educação ambiental comunitária. Anais do $X$ Congresso Nacional de Educação. Curitiba, PR: PUCPR.

Ballesteros, S., Mayas, J., \& Reales, J. M. (2013). Cognitive function in normal aging and in older adults with mild cognitive impairment. Psicothema, 25(1), 18-24. doi:10.7334/psicothema2012.181 
Banhato, E. F. C., \& Nascimento, E. (2007). Função executiva em idosos: Um estudo utilizando subtestes da Escala WAIS-III. PsicoUSF, 12(1), 65-73.

Braver, T. S., \& West, R. (2008). Working memory, executive control, and aging. In F. I. M. Craik, T. A. Salthouse (Eds.), The Handbook of Aging and Cognition. New York, NY: Psychology Press.

Carlson, M. C., Xue, Q. L., Zhou, J., \& Fried, L. P. (2009). Executive decline and dysfunction precedes decline in memory: The women's health and aging study II. The Journals of Gerontology, 1, 110-117. doi: $10.1093 /$ gerona/gln008

Charchat-Fichman, H., Caramelli, P., Sameshima, K., \& Nitrini, R. (2005). Declínio da capacidade cognitiva durante o envelhecimento. Revista Brasileira de Psiquiatria, 27(1), 79-82. doi:10.1590/S1516-44462005000100017

Chaves, M. L., \& Izquierdo, I. (1992). Differential diagnosis between dementia and depression: A study of efficiency increment. Acta Neurologica Scandinavica, 85, 378-382. doi:10.1111/j.1600-0404.1992.tb06032.x

Chen, T. F., Chen, Y. F., Cheng, T. W., Hua, M. S., Liu, H. M., \& Chiu, M. J. (2009). Executive dysfunction and periventricular diffusion tensor changes in amnesic mild cognitive impairment and early Alzheimer's disease. Hum Brain Mapp, 30(11), 3826-3836. doi:10.1002/hbm.20810.

Chen, P., Ratcliff, G., \& Belle. S. H. (2001). Cognitive tests that best discriminate between presymptomatic $A D$ and those who remain nondemented. Neurology, 57(1), 163-164. doi:10.1212/WNL.55.12.1847

Cunha, J. A. (2000). Escalas Wechsler. In J. A. Cunha (Ed.), Psicodiagnóstico-V (pp. 129-602). Porto Alegre, RS: Artes Médicas.

Delano-Wood, L., Abeles, N., Sacco, J. M., Wierenga, C. E., Horne, N. R., \& Bozoki, A. (2008). Regional white matter pathology in mild cognitive impairment: Differential influence of lesion type on neuropsychological functioning. Stroke, 39, 794-799. doi:10.1161/STROKEAHA.107.502534

Diamond, A. (2013). Executive functions. Annual Review of Psychology, 64, 135168. doi: 10.1146/annurev-psych-113011-143750

Gauthier, S., Reisberg, B., Zaudig, M., Petersen, R.C., Ritchie, K., Broich, K., ... Winblad, B. (2006). Mild cognitive impairment. Lancet, 15(1), 1262-1269. doi: 10.1016/S0140-6736(06)68542-5

Gomes, A. L. Z., \& Othero, M. B. (2016). Cuidados paliativos. Estudos Avançados, 30(88), 155-166. doi:10.1590/S0103-40142016.30880011

Green, J. (2000). Neuropsychological evaluation of the older adult: A clinician's guidebook. San Diego, CA: Academic.

Griffith, H. H., Belue, K., Sicola, A., Krzywanski, S., Zamrini, E., Harrell, L., Marson, D. C. (2003). Impaired financial abilities in mild cognitive impairment. Neurology, 60(3), 449-457. doi:10.1212/WNL.60.3.449 
Hamdan, A. C., \& Bueno, O. F. A. (2005). Relações entre controle executivo e memória episódica verbal no comprometimento cognitivo leve e na demência tipo Alzheimer. Estudos de Psicologia (Natal), 10(1), 6371. doi:10.1590/S1413-294X2005000100008

Heaton, R. K., \& Par, S. (2000). WCST-64: Computer version 2 - Research edition (WCST-64: CV2). PAR.

Kaland, N., Smith, L., \& Mortensen, E. L. (2008). Brief report: Cognitive flexibility and focused attention in children and adolescents with Asperger Syndrome or high-functioning autism as measured on the computerized version of the Wisconsin Card Sorting Test. Journal of Autism and Developmental Disorders, 38(6), 1161-1165. doi:10.1007/s10803-0070474-1

Knopman, D. S., Boeve, B. F., \& Petersen, R. C. (2003). Essentials of the proper diagnoses of Mild Cognitive Impairment, Dementia, and major subtypes of Dementia. Mayo Clinic Proceedings, 78, 1290-1308. doi:10.4065/78.10. 1290

Kochhann, R., Varela, J., Lisboa, C. S. M, \& Chaves, M. L. (2010). The Mini Mental State Examination Review of cutoff points adjusted for schooling in a large Southern Brazilian sample. Dementia \& Neuropsychol., 4(1), 35-41. doi:10.1590/S1980-57642010DN40100006

Kongs, S. K., Thompson, L. L., Iverson, G. L., \& Heaton, R. K. (2000). WCST-64: Wisconsin Card Sorting Test-64 card version, professional manual. Odessa, FL: Psychological Assessment Resources.

Kreuz, G., \& Franco, M. H. P. (2017). Reflexões acerca do envelhecimento, problemáticas, e cuidados com as pessoas idosas. Revista Kairós: Gerontologia, 20(2), 117-133. doi:10.23925/2176-901X.2017v20i2p117133

Leon, A., Hahn-Ketter, A., Carrion, C., \& Pliskin, N. A. (2014). The effect of education and diagnosis on the Wisconsin Card Sorting Test performance of a sample of monolingual Spanish-speaking Hispanics. Archives of Clinical Neuropsychology, 29(6), 519. doi:10.1093/arclin/acu038.44

Lino, V. T. S., Pereira, S. R. M., Camacho, L. A. B., Ribeiro Filho, S. T., \& Buksman, S. (2008). Adaptação transcultural da Escala de Independência em Atividades da Vida Diária (Escala de Katz). Cadernos de Saúde Pública, 24(1), 103-112. doi: 10.1590/S0102-311X2008000100010

Lopes, R. M. F, Bastos, A., \& Argimon, I. I. L. (2017). Treino das funções executivas em idosos: Uma revisão sistemática da literatura. Cuadernos de Neuropsicologia, 11(1), 11-29. doi:10.7714/CNPS/11.1.201

Lopes, R. M. F, Ziemniczak, V., Nascimento, R. F. L., \& Argimon, I. I. L. (2015). Funções executivas: A regência de uma orquestra. In I. I. L. Argimon, C. S. Esteves, \& G. W. Wendt (Eds.), Ciclo Vital: Perspectivas contemporâneas em avaliação e intervenção (pp. 71-81). Porto Alegre: EDIPUCRS. 
Malloy-Diniz, L. F., Lasmar, V. A., Gazinelli L. S., Fuentes, D., \& Salgado, J. V. (2007). The Rey Auditory-Verbal Learning Test: Applicability for the Brazilian elderly population. Revista Brasileira de Psiquiatria, 29(4), 324-329. doi: 10.1590/S1516-44462006005000053

Mcguinness, B., Barrett, S. L., Craig, D., Lawson, J., \& Passmore, A. P. (2010). Executive functioning in Alzheimer's disease and vascular dementia. Internacional Journal of Geriatric Psychiatric, 25, 562-568. doi: $10.1002 /$ gps. 2375.

Nascimento, E. (2000). Escala de Inteligência Wechsler para Adultos, terceira edição - WAIS-III. São Paulo, SP: Casa do Psicólogo.

Papalia, D. E., \& Olds, S. W. (2013). Desenvolvimento humano. 12. ed. Porto Alegre, RS: AMGH.

Petersen, R. C., Smith, G. E., Waring, S. C., Ivnik, R. J., Tangalos, E. G., \& Kokmen, E. (1999). Mild cognitive impairment: Clinical characterization and outcome. JAMA Neurology, 56(3), 303-308. doi:10.1001/archneur.56.3.303

Petersen, R. C., Stevens, J. C., Ganguli, M., Tangalos, E. G., Cummings, J. L., \& Dekosky, S. T. (2001). Practice parameter: Early detection of dementia: Mild cognitive impairment (an evidence-based review). Report of the Quality Standards Subcommittee of the American Academy of Neurology. Neurology, 56, 1133-1142. doi:10.1212/WNL.56.9.1133

Petersen, R. C., Caracciolo, B., Brayne, C., Gauthier, S., Jelic, V., \& Fratiglioni, L. (2014). Mild cognitive impairment: A concept in evolution. Journal of Internal Medicine, 275(3), 214-228. doi:10.1111/joim.12190.

Penrod, J., Preston, D. B., Cain, R., \& Starks, M. T. (2003). A discussion of chain referral as a method of sampling hard-to-reach populations. Journal of Transcultural nursing, 4(2), 100-107. doi:10.1177/1043659602250614

Rey, A. (1958). L'Examen Clinique en Psychologie. Paris: Press Universitaire de France.

Ribeiro, E. E. (2008). Tanatologia, vida e finitude. Rio de Janeiro: UERJ, UnATI.

Ribeiro, P. C. C., Oliveira, B. H. D., Cupertino, A. P. F. B., Neri, A. L., \& Yassuda, M. S. (2010). Desempenho de idosos na Bateria Cognitiva CERAD: Relações com variáveis sociodemográficas e saúde percebida. Psicologia: Reflexão e Crítica, 23(1), 102-109. doi:10.1590/S0102-79722010000100013

Royall, D. R., \& Chiodo, L. K. (2004). Executive control and the validity of survey data. International Journal of Geriatric Psychiatry, 19(7), 696-708. doi: 10.1002/gps.1127

Satler, C., Guimarães, L., \& Tomaz, C. (2017). Planning ability impairments in probable Alzheimer's disease patients: Evidence from the Tower of London test. Dementia \& Neuropsychologia, 11(2), 137-144. doi:10.1590/198057642016dn11-020006. 
Silva, J. M. R. (2017). Funcionamento executivo: Estudo comparativo entre controlos saudáveis, défice cognitivo ligeiro e demência de Alzheimer. Dissertação de Mestrado, Faculdade de Psicologia e de Ciências da Educação, Universidade do Porto, Portugal.

Slongo, L. A., Albrecht, C. F., Lavouras, D. F., Esteves, P. S., \& Barcelos, R. H. (2009). A moda para a consumidora da terceira idade. Anais do Encontro Anual da ANPAD, 33, 2009, São Paulo, SP: Associação Nacional dos Cursos de Pós-Graduação em Administração.

Strauss, E., Sherman, E. M. S., \& Spreen, O. (2006). A Compendium of Neuropsychological Tests: Administration, norms and commentary. New York, NY: Oxford University Press.

Sullivan, J. R., Riccio, C. A., \& Castillo, C. R. (2009). Concurrent validity of the Tower Tasks as measures of executive function in adults: A meta-analysis. Applied Neuropsychology, 16(1), 62-75. doi:10.1080/09084280802644 243.

Veras, R. (2009). Envelhecimento populacional contemporâneo: Demandas, desafios e inovações. Revista de Saúde Pública, 43(3), 548-554. doi:10.1590/S0034-89102009005000025.

Wechsler, D. (1997). WAIS-III: Administration and scoring manual. San Antonio: Psychological Corporation.

Winblad, B., Palmer, K., Kivipelto, M., Jelic, V., Fratiglioni, L., Wahlund, L. O., ... Petersen, R. C. (2004). Mild cognitive impairment - beyond controversies, towards a consensus: Report of the International Working Group on Mild Cognitive Impairment. Journal of Internal Medicine, 256(3), 240-246. doi: $10.1111 / \mathrm{j} .1365-2796.2004 .01380 . x$

Sobre as autoras

Cristiane Silva Esteves é psicóloga, especialista em atendimento clínico, com ênfase em Psicanálise pela Universidade Federal do Rio Grande do Sul (UFRGS). Mestre em Psicologia Clínica pela PUC-RS (bolsista CNPq). Doutora em Gerontologia Biomédica pela PUC-RS (bolsista CAPES). crissilvaesteves@gmail.com

Camila Rosa Oliveira é psicóloga pela Universidade Federal do Rio Grande do Sul (UFRGS), Especialização em Terapia Cognitivo-Comportamental pela WP - Centro de Psicoterapia Cognitivo-Comportamental, Mestrado em Psicologia (ênfase cognição humana) pela Pontifícia Universidade Católica do Rio Grande do Sul (PUCRS) e Doutorado em Gerontologia Biomédica da PUCRS. Professora da Escola de Psicologia da IMED - Faculdade Meridional.mila_r3@yahoo.com.br Valéria Gonzatti é psicóloga e possui graduação em Licenciatura em Psicologia pela Universidade Federal do Rio Grande do Sul, mestre e doutoranda em Psicologia pela Pontifícia Universidade Católica do Rio Grande do Sul. valeria.gonzatti@acad.pucrs.br 
Manuela Polidoro Lima é psicóloga, mestre em Ciências da Saúde com ênfase em Oncologia e aluna de doutorado em Psicologia Clínica no Programa de Pós Graduação em Psicologia da Pontifícia Universidade Católica do Rio Grande do Sul (PUCRS). manuela.lima@acad.pucrs.br

Carmen Moret Tatay é professora de ciência cognitiva e metodologia em Valencia (Espanha), na Universidad Católica de Valencia, San Vicente Mártir. Mestre em Neurociência Cognitiva pela Universitat de Valencia e Doutora em Matemáticas Aplicadas na Ciência Cognitiva pela Universitat Politécnica de Valencia. carmenmoret@gmail.com

Irani Iracema de Lima Argimon é psicóloga, possui Mestrado em Educação e Doutorado em Psicologia. É Terapeuta Cognitivo-Comportamental certificada pela Fundação Brasileira de Terapias Cognitivas (FBTC, 2015). argimoni@pucrs.br Tatiana Quarti Irigaray é psicóloga, Doutora e Mestre em Gerontologia Biomédica pela Pontifícia Universidade Católica do Rio Grande do Sul (PUCRS). Tem PósDoutorado em Psicologia pela PUCRS. Atualmente é Coordenadora do Programa de Pós-Graduação em Psicologia da Escola de Ciências da Saúde da PUCRS. tatiana.irigaray@pucrs.br

A contribuição de cada autor pode ser atribuída como se segue: C.O., C.E. e V.G. contribuíram para a conceitualização, investigação e visualização do artigo; C.O. e C.T. foram responsáveis pela análise dos dados da pesquisa e construção das tabelas; M.L., T.I. e I.A. são as responsáveis pela redação final (revisão e edição).

Recebido em: 28/10/2017

Revisado em: 28/02/2018

Aceito em: 26/05/2018 